\section{Editorial: On IRIE Vol. 15}

What is the core of this publication, of a publication in itself? Etymologically the notion 'publication' goes back to the Latin 'publicus' which means 'common', 'of the people/state'. So once executed, the publication, the content, the fruits of the scientific labour needed to produce it belong to everyone, to the people? Far from it! According to applicable law and common moral standards the (intellectual) property fully remains with the originators. It is only accessible for everyone (in the case of our journal even accessible for free) and everybody can make use of it; but only according to the very defined rules of the scientific discourse, i.e. citing and referring.

Scandals about the illegal obtaining of doctoral degrees by extensive plagiarism in Germany have reminded us of this specific aspect of the rules of the scientific game. So what is 'common' with regards to this publication and what exactly is made public by the publication?

Interesting questions but yet a misleading approach for the subject of this issue. The scientific discourse and its standards of publishing and citing are not and have never been the template of what is driving the largest communication machinery ever: the world wide web. In fact, the guiding principle of the underlying technology $\mathrm{html}$ and the internet protocol - was to realize an unprecedented ease of referring (i.e. linking) from one publication to another explicitly leaving out the scientific standards of citation. This informal sharing of information is fundamentally woven into what we call most appropriate: 'the net' - the loose coupeling of communication shared by anyone with anyone.

In information ethics though 'sharing' has been discussed so far only implicitly in terms of privacy, intellectual property, secrecy, security and freedom of speech. But not only that libraries have been at least challenged by search engines; also recent developments of a second order like the encyclopedia project Wikipedia, the emergence of social networks like facebook or disclosure platforms like WikiLeaks have shown that there is a need to go beyond the scientific habits and legal standards of sharing knowledge and distributing information to understand and govern the communicative space and exchange of information made possible by the internet and its respective platforms.

So, has sharing of information a special virtue in the information society? How are choices of sharing or withholding of information justified? Is sharing subversive of the new global information regime, or an integral aspect of it?

This issue brings together contributions towards an ethics of sharing that embed the new technological potentialities linking them to their actual social impact. In our understanding, information ethics "deals with ethical questions in the field of digital production and reproduction of phenomena and processes such as the exchange, combination and use of information." So, the task of developing an ethics of sharing is both descriptive - helping us to understand the contemporary complexities of the ethics of exchanging information as it emerges from using digital technologies across a global range of social and cultural contexts - as well as normative - helping us to address blind-spots and clarifying possible ethical frameworks to address unresolved issues regarding these practices.

And what do we and should we finally do with the truly impressive contributions gathered here to provide answers to the above named questions and guidelines for the outlined task? We share them with you leaving them to your appropriate use - whatever you may make out of it.

Yours,

the editors.

P.S. Please take also note that we added another article to the last issue (No. 14) on "Teaching Information Ethics". Bernardo Sorj and Mauricio Lissovsky examined the use of the internet in Brazilien Public Schools and what it needs to make appropriate use of the costly equipment and the new technologies becoming more and more available to teachers and pupils in the country. Unfortunately we became aware of their most interesting observations and conclusions only a few weeks after the copy date of the last issue. But not living in the Gutenberg Galaxy anymore this is not an ultimate criterion of exclusion but only a few more lines of html added to the website. 